\title{
Testing of the accuracy dependency of prismless distance measurement on the beam incidence angle
}

\author{
Pavel Třasák, Martin Štroner, Václav Smítka, Rudolf Urban \\ Department of Special Geodesy \\ Faculty of Civil Engineering \\ Czech Technical University in Prague \\ pavel.trasak@fsv.cvut.cz
}

Keywords: prismless distance measurement, accuracy, testing, incident angle

\begin{abstract}
The article assesses the precision development of distance measurement using prismless distance meters, both in relation to the changing length of the measured distance and the changing incidence angle of the distance meter's beam. The article presents the design and performance of an original experiment, the characteristics of instruments used, statistical evaluation of experimental data and formulation of conclusions on the precision rate of distance measurement using prismless instruments.
\end{abstract}

\section{Introduction}

The problems falling under research plan VZ 1 "Reliability, optimization and durability of building materials and constructions", partial task "Geodetic monitoring ensuring the reliability of structures", also involve testing surveying measuring instruments, which is a key part of metrological safeguarding of instruments and aids used in on-site surveying measurements.

Measurements using prismless distance meters are becoming increasingly more common in surveying practice allowing the measurement of points directly on structures eliminating the need for reflective beacons (reflective foils, corner cube prisms). Their principal advantages include time economies and frequently also considerable cost economies in the case of poor accessibility of measured points. The measurement of points situated on rock walls, in mines or on façades of buildings may serve as an example.

The precision rate of measurements using prismless distance meters tends to be lower than in measurements applying reflective prisms or foils, which, however, does not pose a problem in many jobs. To utilize this technology with adequate effectiveness, it is advisable to know the characteristics of the measurement. It is a generally known and recognized fact that the precision of measurements made with this type of distance meters falls with the growing deflection from the normal incidence onto the measured surface. The article describes a measuring experiment whose objective is to confirm or disprove the above-mentioned fact about a systematically falling precision of measured distances.

Due to the fact that different manufacturers of measuring instruments may use different electro-optical systems of determining measured distances, three different instruments commonly applied in surveying practice were used for the experiment - total stations equipped with prismless distance meters and also a terrestrial laser scanner. 


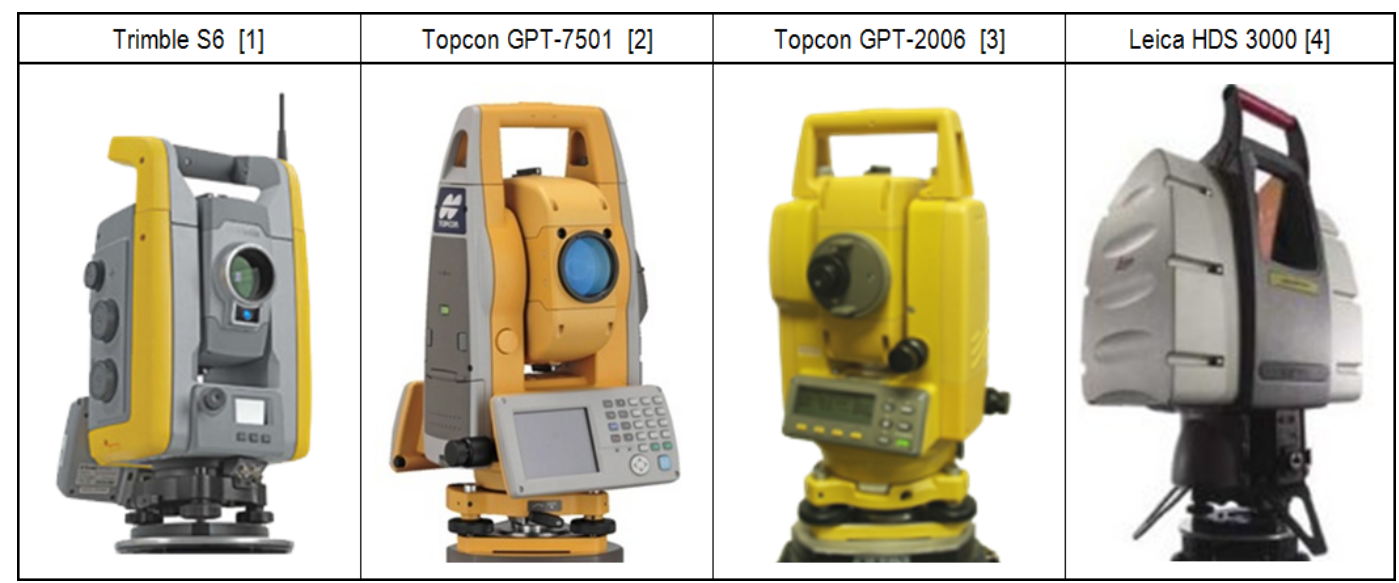

Figure 1: Used instruments

\section{Instruments and aids used}

The testing was performed with instruments and measurement aids currently available at the Department of Special Geodesy at Faculty of Civil Engineering, CTU in Prague.

\section{Tested measuring instruments}

As mentioned above, the total of 4 measuring instruments were used during the measuring experiment (3 total stations, 1 terrestrial laser scanner). For the reason of preserving the maximum objectivity of the experiment, the measuring instruments were selected to cover the most varied characteristics possible. The choice of instruments was conditioned on their diversity in terms of the instrument design (different manufacturers), technological differences (varied age of instruments) and in terms of varied precision rates of implemented prismless distance meters. References to literature containing detailed information on the instruments are displayed in Fig. 1, while precision rate characteristics are presented in Tab. 1.

\begin{tabular}{|c|r|r|r|r|}
\hline & \multicolumn{1}{|c|}{ Trimble S6 } & Topcon GPT-7501 & Topcon GPT-2006 & Leica HDS 3000 \\
\hline \hline$\sigma_{\phi}$ & $0.3 \mathrm{mgon}$ & $0.3 \mathrm{mgon}$ & $2.0 \mathrm{mgon}$ & $3.8 \mathrm{mgon}$ \\
\hline$\sigma_{z}$ & $0.3 \mathrm{mgon}$ & $0.3 \mathrm{mgon}$ & $2.0 \mathrm{mgon}$ & $3.8 \mathrm{mgon}$ \\
\hline$\sigma_{D H}$ & $1 \mathrm{~mm}+1 \mathrm{ppm}$ & $2 \mathrm{~mm}+2 \mathrm{ppm}$ & $3 \mathrm{~mm}+2 \mathrm{ppm}$ & \\
\hline$\sigma_{D B}$ & $3 \mathrm{~mm}+2 \mathrm{ppm}$ & $5 \mathrm{~mm}$ & $\begin{array}{r}10 \mathrm{~mm}(<25 \mathrm{~m}) \\
3 \mathrm{~mm}+2 \mathrm{ppm}(>25 \mathrm{~m})\end{array}$ & $4 \mathrm{~mm}(<50 \mathrm{~m})$ \\
\hline
\end{tabular}

Table 1: Characteristics of selected measuring instruments, where

$\sigma_{\phi}$ is standard deviation of measured horizontal directions, $\sigma_{z}$ of measured zenithal distances, $\sigma_{D H}$ of distances measured by a prism distance and $\sigma_{D B}$ of distances measured by a prismless distance meter

\section{A device simulating a reflective surface}

The target surface allowing the reflection of a distance meter's beam of electromagnetic rays used was a wooden board with dimensions of $420 \mathrm{~mm}$ (height) $\times 320 \mathrm{~mm}$ (width). The 


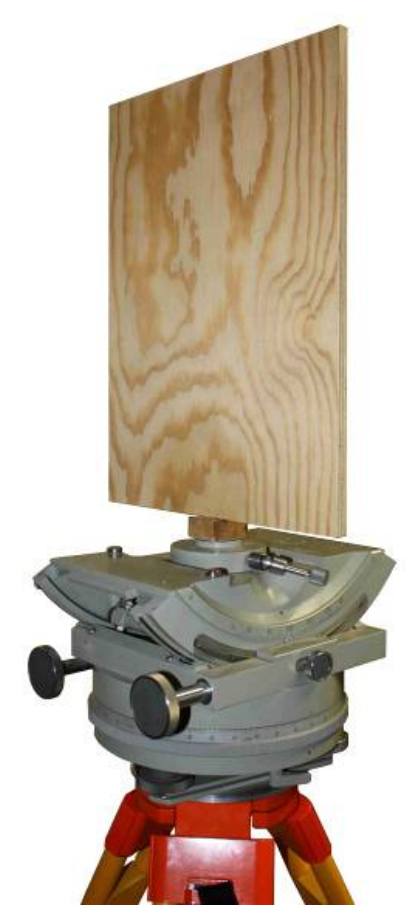

Figure 2: A target wooden board fixed onto the photogrammetric camera carrier and placed on a surveyor's tripod

board was fixed onto the carrier of a terrestrial photogrammetric camera (Fig. 2), which, in combination with a surveyor's tripod, allows precise positioning of the vertical axis of rotation of the target board above the target point (baseline point, see below) and precise turning of the reflective surface.

\section{Description of the experiment}

The measuring experiment was planned to test measurements made by prismless distance meters of selected instruments under various incidence angles of the distance meter's laser beam to the surface of the measured object (wooden reflective board). Due to the fact that different behaviour of measuring instruments during the measurement of different distances may be expected, the measurement precision was tested not only in relation to the incidence angle of the distance meter's beam to the reflective surface, but also in relation to the length of the measured distance.

The site selected for the performance of the measuring experiment were the premises of the ground-floor technological storey of the building of the Faculty of Civil Engineering, CTU in Prague where all-day unchangeable conditions may be expected affecting the use of a prismless electronic (laser) distance meter (or affecting the transmission of the distance meter's beam of electromagnetic rays respectively), i.e. all-day constant temperature, atmospheric pressure and air humidity, and, further, a uniform unchangeable light exposure of the whole measured space.

In the first phase of the experiment, the baseline was set out with a precision of 1 centimetre, and the points for the positioning of the measured surface were located at distances 
of $5 \mathrm{~m}, 10 \mathrm{~m}, 20 \mathrm{~m}, 40 \mathrm{~m}$ from the measuring station $\mathrm{S}$ (the measuring instrument's centre). As experimental data processing does not consider working with actual deflections of measured distances, but only with sample standard deviations determined from repetitive measurements, 1-cm precision in setting out the baseline is quite sufficient.

Both the measuring instrument (this generally applies to all selected instruments) and the reflective board (together with the photogrammetric camera carrier) were fixed onto surveyor's tripods and located above the baseline points at an approximate height of ca $1.2 \mathrm{~m}$. The turning of the target board for the experiment was selected in an interval of 0 to 90 gon. While gradually turning the surface in 15-gon steps, the total of 7 board turning positions were obtained ( 0 gon, 15 gon, 30 gon, 45 gon, 60 gon, 75 gon, 90 gon).

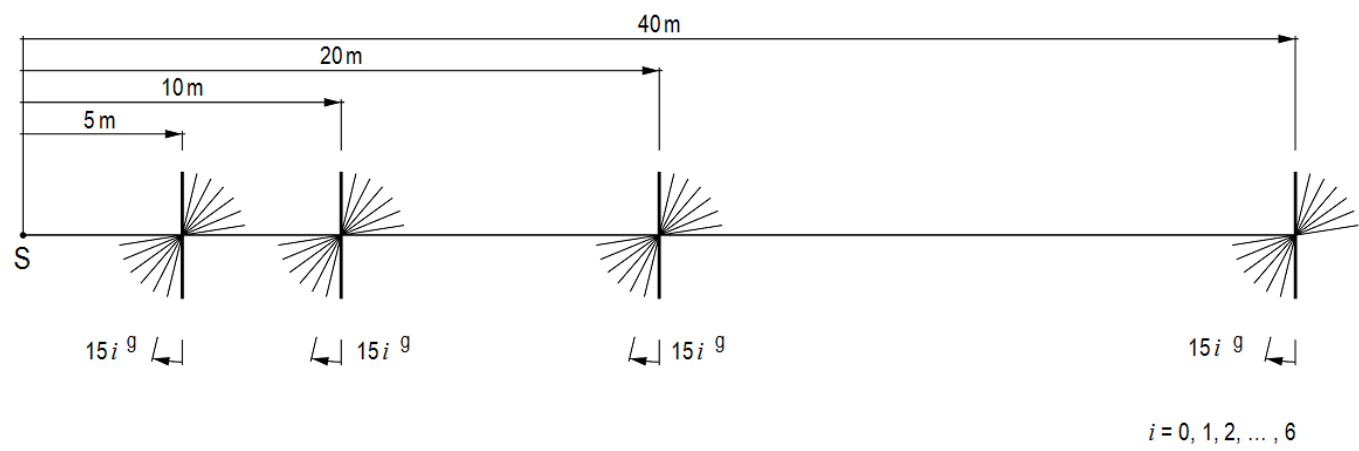

Figure 3: Baseline diagram

During the whole time of the experiment, the tested instrument was placed at the initial point of the baseline, and the tripod with the reflective target board was gradually centred and levelled over individual baseline points. For each configuration set-up, i.e. for each distance ( 4 distances in all) and for each turning of the reflective board ( 7 turnings in all), repetitive measurements of the horizontal distance were performed using a prismless electronic distance meter.

\section{Specification of the number of repetitive distance measurements}

The number $\mathrm{n}$ of repetitive measurements of the distance was specified based on the consideration of the magnitude of the standard deviation of the sample standard deviation of the measured distance $\sigma_{s}[5]$, which is defined by the relation

$$
\sigma_{s}=\frac{\sigma}{\sqrt{2(n-1)}},
$$

where $\sigma$ is the standard deviation of the distance. Based on the previously formulated condition that the standard deviation $\sigma_{s}$ may maximally equal $10 \%$ of the magnitude of the standard deviation $\sigma$ the range of random sampling equals

$$
\sigma \frac{1}{\sqrt{2(n-1)}}=0.1 \sigma \Rightarrow n=51 .
$$

For the total number of 7 different positions of partial turning of the target board ( 0 gon, 15 gon, 30 gon, 45 gon, 60 gon, 75 gon, 90 gon) and 51 repetitive measurements, the total of 357 distances were to be measured at each target point of the baseline. For the whole baseline 
(4 different target points), the total number of distances measured by one measuring instrument equals 1428 (28 random samples). In the whole experiment considering 4 instruments, the total of 5712 distances are measured.

\section{Assessment and results of the experiment}

\section{Processing procedure}

As mentioned above, the objective of the experiment was to assess the precision development of distance measurements made by a prismless distance meter, both in relation to the growing length of the measured distance and the growing turning of the reflective surface. The principal characteristic describing the distance meter's accuracy considered in this case is the sample standard deviation determined from repetitive measurements of a distance in a certain configuration (at a certain distance and partial turning of the reflective surface), i.e. the sample standard deviation of a random sample with a size of 51 values. This deviation is governed by the relation

$$
s=\sqrt{\frac{1}{n-1} \sum_{i=1}^{n}\left(x_{i}-\bar{x}\right)^{2}},
$$

where $n$ is the sample size, $x_{i}$ is the $i$-th measured distance and is the sample mean of measured distances.

The final result of data processing within the experiment is the assessment of input random samples $(4 \times 28$ in total), determination of sample standard deviations and assessment of whether their development within a changing configuration is random or whether it is influenced by significant effects participating in the reduced accuracy of distances measured by prismless distance meters. Experimental data is processed using the following procedure:

1. Testing of outliers in random samples and their potential elimination.

2. Verification of normality of random samples.

3. Homogeneity assessment of random samples.

4. Setting of the confidence interval for the standard deviation of a measured distance.

5. Regression analysis, setting of a linear change in the standard deviation of a measured distance.

6. Graphic interpretation of accuracy evolution of a measured distance.

\section{Elimination of outliers}

In repetitive measurements of distances using an electronic distance meter, potential effects of sporadic gross measurement errors are presumed (caused e.g. by a potential sudden fluctuation of natural conditions in the vicinity of the measured distance). This leads to the occurrence of outlying measurement values in random samples of measured distances. It is advisable to eliminate outliers from the random samples during their processing thus enhancing the objectivity of the results of evaluated data. 
Based on an assumption that random samples belong to the population with a normal probability distribution and sample sizes are relatively large, the outliers to be eliminated were detected on the basis of Grubbs' test [6].

Testing on the $5 \%$ significance level detected individual outliers of measured distances (in the most unfavourable case, 3 outliers were eliminated from a random sample).

\section{Verification of normality of random samples}

Random samples of repetitive distance measurements are presumed to belong to the population with a normal probability distribution. To confirm this assumption, experimental data are subjected to testing using D'Agostino's K2 test [7].

The result of testing on the $5 \%$ significance level confirmed the normality of all tested random samples.

\section{Homogenity assessment of random samples}

The verification of homogeneity of random samples of measured distances is one of the possibilities allowing the assessment of the effect of the configuration (the effect of the length of the measured distance and the turning of the reflective surface) on the accuracy rate of a distance measured by a prismless distance meter. The principle of this method consists in the application of statistical homogeneity tests. These tests work with groups $\mathrm{k}$ of random samples and verify the null hypothesis that the variances of the populations $\sigma_{i}^{2}$, to which random samples belong, are equal to each other

$$
\begin{aligned}
& H_{0}: \sigma_{1}^{2}=\sigma_{2}^{2}=\ldots=\sigma_{g}^{2} \\
& H_{1}: \sigma_{1}^{2} \neq \sigma_{2}^{2} \neq \ldots \neq \sigma_{g}^{2} .
\end{aligned}
$$

Hence, created groups of random samples of measured distances allow testing whether the sample standard deviations of measured distances $s_{i}$ (or sample variances $s_{i}^{2}$ respectively) are in correspondence with the joint standard deviation of the distance $\sigma$ (or the variances of the population $\sigma^{2}$ respectively). Thus, provided all samples corresponding to the same distance (4 groups with 7 samples each) are sorted out, and provided the null hypothesis is not rejected during the testing of these groups, it may be verified that the accuracy rate of the measured distance does not rely on the turning of the reflective surface (i.e. on the incidence angle of the distance meter's beam).

Based on the assumptions about the normality of random samples, Bartlett's test was selected for the testing of homogeneity. The testing criterion of Bartlett's test testing the group $k$ of random samples is the variable $\mathrm{B}$, which is defined by the expression

$$
B=\frac{\left[(N-1) \ln s_{c}^{2}-\sum_{i=1}^{k}\left(n_{i}-1\right) \ln s_{i}^{2}\right]}{C},
$$

where the constant $\mathrm{C}$ equals

$$
C=\frac{1+\left(\sum_{i=1}^{k} \frac{1}{n_{i}-1}-\frac{1}{N-k}\right)}{3(k-1)}
$$


$s_{i}^{2}$ is the sample variance of the $i$-th sample of measured distances with a range $n_{i}$

$$
s_{i}^{2}=\frac{1}{n_{i}-1} \sum_{j=1}^{n}\left({ }^{i} x_{j}-{ }^{i} \bar{x}\right)^{2}, \quad i=1,2, \ldots, k,
$$

and $s_{c}^{2}$ is the pooled estimation of the variance expressed in the form

$$
s_{c}^{2}=\frac{1}{N-1} \sum_{i=1}^{k} \sum_{j=1}^{n_{i}}\left({ }^{i} x_{j}-{ }^{i} \bar{x}\right)^{2}, \quad N=\sum_{i=1}^{k} n_{i} .
$$

Assuming the validity of the null hypothesis and the condition of the minimum number of tested samples $n_{i} \geq 6$, the variable $B$ roughly has the distribution $\chi^{2}$.

The acceptance or rejection of the null hypothesis about the equality of variances of populations is assessed on the level of significance

$$
P\left(B>\chi_{\alpha,(k-1)}^{2}\right)=\alpha=0.05,
$$

where $\chi_{2 \alpha,(k-1)}^{2}$ is the critical value of the distribution $\chi^{2}$ with $(k-1)$ degrees of freedom.

Provided the value of the testing criterion $B$ exceeds the critical value $\chi_{2 \alpha,(k-1)}^{2}$, the null hypothesis on the level of significance $\alpha$ is rejected and homogeneity of samples is not proved.

Besides the decision on the rejection or confirmation of the null hypothesis on the selected level of significance $\alpha$, the testing also results in the determination of the $p$-value, i.e. the probability at which the testing criterion equals the critical value

$$
P\left(B=\chi_{p,(k-1)}^{2}\right)=p .
$$

The determined $p$-value describes the limit level of significance, i.e. the maximum probability of the rejection of the null hypothesis, despite its validity.

The results of testing the homogeneity of groups of random samples of measured distances are displayed in Tab. 2.

The results of testing the groups of random samples in which the null hypothesis was not rejected on the $5 \%$ level of significance during the testing of homogeneity, i.e. in which the effect of the distance meter's beam incidence angle on the accuracy rate of the distance measured by a prismless distance meter was not proved on the selected level of significance, are written in bold in the table above.

\section{Confidence interval for the standard deviation of a measured distance}

Another method used for the investigation of the effect of turning the reflective surface on the precision of a measured distance is the method based on the interval estimation of the standard deviation of a measured distance. The method consists in the creation of a two-sided $95 \%$ confidence interval for the standard deviation of the measured distance $\sigma$. Assuming that the random sample of measured distances (serving for the construction of the confidence interval) is taken from the population with a normal probability distribution, the $95 \%$ confidence interval is set as

$$
P\left(\sqrt{\frac{(n-1) s^{2}}{\chi_{1-\alpha / 2, n-1}^{2}}} \leq \sigma \leq \sqrt{\frac{(n-1) s^{2}}{\chi_{\alpha / 2, n-1}^{2}}} \leq\right)=1-\alpha=0.95,
$$


Trasak, P. et al: Testing of the ACCuracy Dependency of Prismless Distance MEASUREMENTS ON THE BEAM INCIDENCE ANGLE

Trimble S6

\begin{tabular}{|c|c|c|c|}
\hline $\begin{array}{c}\text { Distance } \\
{[\mathrm{m}]}\end{array}$ & $C$ & $\chi_{0.05,6}^{2}$ & $\begin{array}{c}p \text {-value } \\
{[\%]}\end{array}$ \\
\hline \hline 5 & 45.452 & & 0.0 \\
10 & 22.737 & 12.592 & 0.1 \\
20 & 15.019 & & 2.0 \\
40 & 94.285 & & 0.0 \\
\hline
\end{tabular}

Topcon GPT-2006

\begin{tabular}{|c|c|c|c|}
\hline $\begin{array}{c}\text { Distance } \\
{[\mathrm{m}]}\end{array}$ & $C$ & $\chi_{0.05,6}^{2}$ & $\begin{array}{c}p \text {-value } \\
{[\%]}\end{array}$ \\
\hline \hline 5 & 45.452 & & 0.7 \\
10 & 22.737 & 12.592 & 80.6 \\
20 & 15.019 & & 3.6 \\
40 & 94.285 & & 23.1 \\
\hline
\end{tabular}

Topcon GPT-7501

\begin{tabular}{|c|c|c|c|}
\hline $\begin{array}{c}\text { Distance } \\
{[\mathrm{m}]}\end{array}$ & $C$ & $\chi_{0.05,6}^{2}$ & $\begin{array}{c}p \text {-value } \\
{[\%]}\end{array}$ \\
\hline \hline 5 & 9.305 & & 15.7 \\
10 & 7.599 & 12.592 & 26.9 \\
20 & 11.881 & & 6.5 \\
40 & 69.255 & & 0.0 \\
\hline
\end{tabular}

Leica HDS 3000

\begin{tabular}{|c|c|c|c|}
\hline $\begin{array}{c}\text { Distance } \\
{[\mathrm{m}]}\end{array}$ & $C$ & $\chi_{0.05,6}^{2}$ & $\begin{array}{c}p \text {-value } \\
{[\%]}\end{array}$ \\
\hline \hline 5 & 14.442 & & 2.5 \\
10 & 1.792 & 12.592 & 93.8 \\
20 & 8.384 & & 21.1 \\
40 & 15.096 & & 2.0 \\
\hline
\end{tabular}

Table 2: Results of Bartlett's test of homogeneity of samples

where $1-\alpha$ is the confidence coefficient, $n$ is the sample size, $s$ is the sample standard deviation of a measured distance and $\chi_{1-\alpha / 2,(n-1)}^{2}\left(\right.$ resp. $\left.\chi_{\alpha / 2,(n-1)}^{2}\right)$ is the value of the distribution $\chi^{2}$ with $(n-1)$ degrees of freedom.

The principle of assessing the configuration effect on the precision rate of distance measurement consists in the sorting out of random samples into individual groups according to the length of measured distances (see sorting into groups in par. "Homogenity assessment of random samples"), determination of confidence intervals for the standard deviation of a distance and assessment whether the confidence intervals in individual groups overlap and, therefore, there exists a common interval in which a standard deviation of a distance common for the whole group is found. In the case that such an interval is found, we may claim that random samples are in correspondence with the same standard deviation of a distance and that the precision rate of distance measurement is not affected by a change in turning the reflective surface at a certain measured distance.

The occurrence of intervals of a potential common standard deviation was studied only graphically, and the results are displayed in Fig. 4.

The results in the figure above correspond to a greater part to the results obtained during the testing of homogeneity of samples (see par. "Homogenity assessment of random samples").

\section{Setting a linear change in the standard deviation of a measured distance}

With respect to the linearly growing incidence angle of the distance meter's beam to the reflective target surface, according to a speculated, generally recognized opinion (see par. "Introduction"), a linear or another monotonous growth pattern of the standard deviation of a measured distance $\sigma$ may be assumed. Based on this assumption, the dependence between the incidence angle of the distance meter's beam and the accuracy rate of a distance measured by a prismless distance meter may be investigated using the regression analysis method. The 

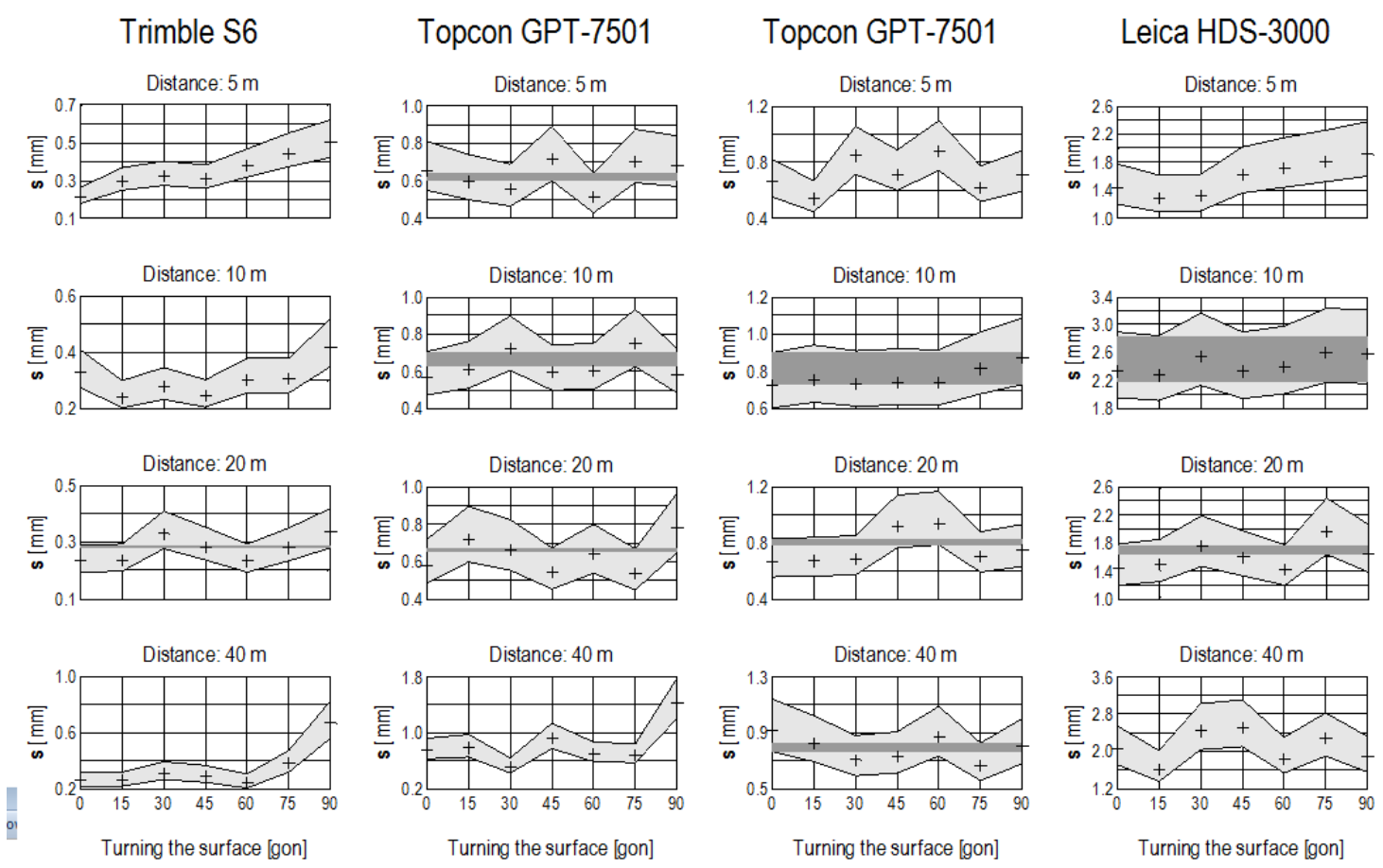

$95 \%$ confidence interval for the standard deviation of the measured distance Interval of a potential common standard deviation of measured distance Sample standard deviation of measured distance

Figure 4: 95\% confidence interval for the standard deviation of a distance

principle of this method consists in the plotting of a regression line

$$
s=a \omega+b
$$

through a set of sample standard deviations of the distance $s$ and assessment of the significance of the slope a of this line representing a linear change in the sample standard deviation $\mathrm{s}$ in relation to the changing angle of the turning of the reflective surface $\omega$. Using the least squares method the estimation of the slope of the regression line in the form

$$
\hat{a}=\frac{\sum_{i=1}^{n}\left(\omega_{i}-\bar{\omega}\right) s_{i}}{\sum_{i=1}^{n}\left(\omega_{i}-\bar{\omega}\right)^{2}}
$$

where si is the sample standard deviation and $\omega_{i}$ is the incidence angle of the distance meter's beam for the $\mathrm{i}$-th turning of the reflective surface, $n$ is the number of turnings of the reflective surface $(n=7)$ and $\bar{\omega}$ is the sample mean incidence angle of the distance meter's beam.

The significance of a change in the sample standard deviation s in relation to a change in the turning of the reflective surface $\omega$ may be assessed on the basis of the statistical hypothesis test where the null hypothesis of the null slope of a regression line is investigated

$$
\begin{aligned}
& H_{0}: a=0, \\
& H_{1}: a \neq 0 .
\end{aligned}
$$


The testing criterion is defined by the relation

$$
t=\frac{|\hat{a}|}{s_{\hat{a}}}
$$

where sâ is the estimation of the standard deviation of the regression line slope estimation

$$
S_{\hat{a}}=\frac{S_{r}}{\sqrt{\sum_{i=1}^{n}\left(\omega_{i}-\bar{\omega}\right)^{2}}}
$$

where $s_{r}$ is the residual standard deviation expressed in the form

$$
S_{r}=\sqrt{\frac{\sum_{i=1}^{n}\left(S_{i}-\left(\hat{a} \omega_{i}+\hat{b}\right)\right)^{2}}{n-2}} .
$$

The acceptance or rejection of the null hypothesis of the null slope of the regression line is assessed on the level of significance

$$
P\left(t>t_{\alpha / 2, n-2}\right)=\alpha=0.05
$$

where $t_{\alpha / 2, n-2}$ is the critical value of Student's distribution $\mathrm{t}$ with $n-2$ degrees of freedom.

If the value of the testing criterion $\mathrm{t}$ exceeds the critical value $t_{\alpha / 2, n-2}$, the null hypothesis on the level of significance $\alpha$ is rejected and the null value of the slope of the regression line is not proved.

$P$ - the value for the test of the slope of the regression line equals the probability

$$
P\left(t>t_{p / 2, n-2}\right)=p .
$$

The resulting values of changes in sample standard deviations, together with the results of the testing, are shown in Tab. 3 .

The results of testing where the null hypothesis was not rejected on the $5 \%$ level of significance during the testing, i.e. in which a null linear change in the sample standard deviation was proved on the selected level of significance, are written in bold in the table above. As the results imply, the non-effect of the unfavourable trend in the accuracy evolution of prismless distance measurement was proved in the absolute majority of cases.

\section{Graphic interpretation of precision development of a measured distance}

To simplify the interpretation of the precision development of prismless measurement of distances in relation to a selected measurement configuration (see above), the resulting sample standard deviations of the distance $\mathrm{s}$ determined from repetitive measurements were displayed in three-dimensional graphs, both in relation to the growing measured distance and the growing incidence angle of the distance meter's beam (i.e. turning of the reflective surface). To get a more illustrative picture of the precision development of measured distances, the set of sorted out standard deviations was approximated on a plain surface (Fig. 5). The approximation was performed using the bicubic interpolation method. 
Trasak, P. et al: Testing of the ACCuracy Dependency of Prismless Distance MEASUREMENTS ON THE BEAM INCIDENCE ANGLE

Trimble S6

\begin{tabular}{|c|c|c|c|c|c|}
\hline $\begin{array}{c}\text { Distance } \\
{[\mathrm{m}]}\end{array}$ & $\begin{array}{c}\hat{a} \\
{[\mu \mathrm{m} / \text { gon }]}\end{array}$ & $\begin{array}{c}S_{\hat{a}} \\
{[\mu \mathrm{m} / \text { gon }]}\end{array}$ & $t$ & $t_{0.025,5}$ & $\begin{array}{c}p \text {-value } \\
{[\%]}\end{array}$ \\
\hline \hline 5 & 2.9 & 0.4 & 8.119 & & 0.0 \\
10 & 1.0 & 0.7 & 1.423 & 2.571 & 21.4 \\
20 & 0.7 & 0.5 & 1.396 & & 22.2 \\
40 & 3.3 & 1.4 & 2.384 & & 6.3 \\
\hline
\end{tabular}

Topcon GPT-7501

\begin{tabular}{|c|c|c|c|c|c|}
\hline $\begin{array}{c}\text { Distance } \\
{[\mathrm{m}]}\end{array}$ & $\begin{array}{c}\hat{a} \\
{[\mu \mathrm{m} / \text { gon }]}\end{array}$ & $\begin{array}{c}S_{\hat{a}} \\
{[\mu \mathrm{m} / \text { gon }]}\end{array}$ & $t$ & $t_{0.025,5}$ & $\begin{array}{c}p \text {-value } \\
{[\%]}\end{array}$ \\
\hline \hline 5 & 0.6 & 1.1 & 0.572 & & 59.2 \\
10 & 0.5 & 1.0 & 0.485 & 2.571 & 64.8 \\
20 & 0.5 & 1.2 & 0.404 & & 70.3 \\
40 & 4.8 & 3.5 & 1.394 & & 22.2 \\
\hline
\end{tabular}

Topcon GPT-2006

\begin{tabular}{|c|c|c|c|c|c|}
\hline $\begin{array}{c}\text { Distance } \\
{[\mathrm{m}]}\end{array}$ & $\begin{array}{c}\hat{a} \\
{[\mu \mathrm{m} / \text { gon }]}\end{array}$ & $\begin{array}{c}S_{\hat{a}} \\
{[\mu \mathrm{m} / \text { gon }]}\end{array}$ & $t$ & $t_{0.025,5}$ & $\begin{array}{c}p \text {-value } \\
{[\%]}\end{array}$ \\
\hline \hline 5 & 0.8 & 1.7 & 0.501 & & 63.8 \\
10 & 1.4 & 0.5 & 2.966 & 2.571 & 3.1 \\
20 & 1.4 & 1.5 & 0.927 & & 39.6 \\
40 & -1.1 & 1.2 & 0.982 & & 37.1 \\
\hline
\end{tabular}

Leica HDS 3000

\begin{tabular}{|c|c|c|c|c|c|}
\hline $\begin{array}{c}\text { Distance } \\
{[\mathrm{m}]}\end{array}$ & $\begin{array}{c}\hat{a} \\
{[\mu \mathrm{m} / \text { gon }]}\end{array}$ & $\begin{array}{c}S_{\hat{a}} \\
{[\mu \mathrm{m} / \text { gon }]}\end{array}$ & $t$ & $t_{0.025,5}$ & $\begin{array}{c}p \text {-value } \\
{[\%]}\end{array}$ \\
\hline \hline 5 & 6.9 & 1.4 & 4.983 & & 0.4 \\
10 & 2.9 & 1.3 & 2.214 & 2.571 & 7.8 \\
20 & 3.1 & 2.3 & 1.348 & & 23.6 \\
40 & 0.4 & 4.6 & 0.088 & & 93.4 \\
\hline
\end{tabular}

Table 3: Assessment of linear changes in sample standard deviations

\section{Conclusion}

In the branch of engineering geodesy and laser scanning, it is currently generally recognized that the precision rate of distance measurement obtained by a prismless distance meter falls with the growing incidence angle of the distance meter's beam to the reflective surface of the target object. Therefore, a measuring experiment was performed to confirm or disprove the validity of this opinion.

The results reached during the experiment (displayed in Tab. 2, Tab. 3 and in Fig. 4, Fig. 5) lead to a conclusion that the incidence angle of the distance meter's beam to the reflective surface has no effect on the standard deviation of a distance measured by a prismless distance 

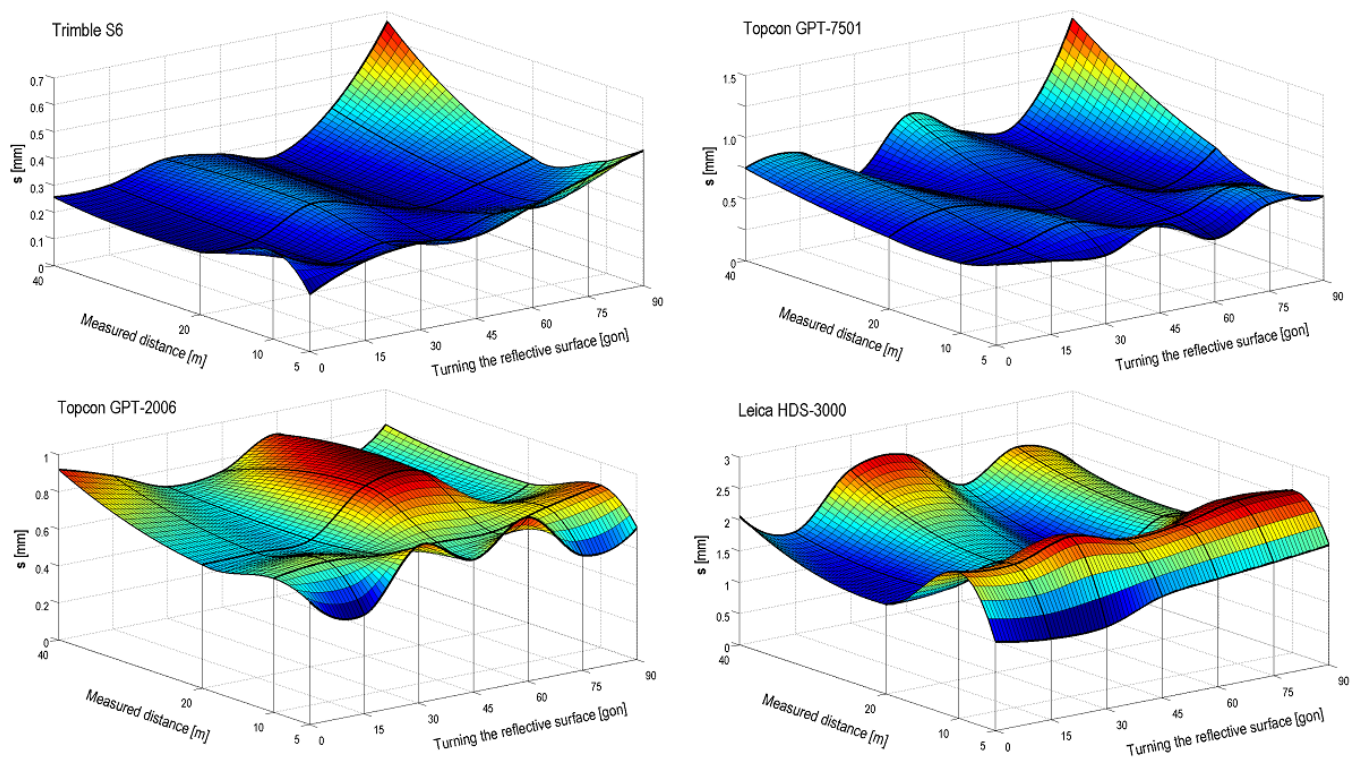

Figure 5: Graphic display of precision development of distance measurement by a prismless distance meter

meter. The values of sample standard deviations of measured distances (determined from repetitive measurements) show random fluctuations and do not imply any systematic drop in the accuracy rate of prismless measurement of distances. An exception is the case of a rapid growth in the sample standard deviation of a distance at a measured distance of $40 \mathrm{~m}$ and turning of the reflective surface by 90 gon using Trimble S6 and Topcon GPT-7501 measuring instruments. This growth, however, cannot be included in the final conclusions of the experiment as it represents an extremely unfavourable case of distance measurement. Assuming a cone-shaped divergence of the distance meter's laser beam, the magnitude of the laser track at a distance of $40 \mathrm{~m}$ is so large and the width of the tested reflective surface in the direction of a falling beam at a turning by 90 gon is so small that it cannot be guaranteed that the whole distance meter's beam will be reflected from this board. The results of measurement in this case are highly untrustworthy.

No significant systematic (linear) deterioration in the accuracy rate of prismless distance measurement in relation to the growing incidence angle of the distance meter's beam to the reflective target surface was proved on the basis of regression analysis of measured data.

Besides the principal objective of the experiment, another new finding was made while comparing the values of sample standard deviations of measured distances with corresponding standard deviations of measurement stated by the instrument manufacturers ( $\sigma_{D B}$, see Tab. 1). These sample standard deviations describe the random component of the total standard deviation $\sigma_{D B}$ and are significantly lower in value than the standard deviations. We may, therefore, assume that the manufacturers consider a relatively high systematic component of the standard deviation of a distance, which, of course, is not manifested in repetitive distance measurements.

To conclude, we may state that the generally recognized opinion of the effect of the distance meter's beam incidence angle (turning of the target reflective surface) on the accuracy rate 
of distances measured by a prismless distance meter is not correct for distances measured in an interval of $0 \mathrm{~m}-40 \mathrm{~m}$. This effect was not manifested during the performance of the measuring experiment.

The article was written with support from research plan MSM 6840770001 "Reliability, optimization and durability of building materials and constructions", partial task "Geodetic monitoring ensuring the reliability of structures".

\section{References}

1. Corporate literature for instrument Trimble S6 (in Czech) ${ }^{1}$ 2010-05-15.

2. Corporate literature for instrument Topcon GPT - 7501 (in Czech). http://obchod. geodis.cz/geo/gpt-7500. 2010-05-15.

3. Štroner, M. - Suchá, J. - Pospíšil, J.: Verification of Characteristics of Total Stations TOPCON GPT-2006 - Part 1 (in Czech). Stavební obzor. 2007, Vol. 16, No. 2, pp. 45-48. ISSN 1210-4027.

4. Corporate literature for instrument Leica HDS 3000. http://hds. leica-geosystems . com/en/5574.htm. 2010-05-15.

5. Böhm, J. - Radouch, V. - Hampacher, M.: Theory of Errors and Adjustment Calculus (in Czech), 2nd edition, Praha, Geodetický a kartografický podnik 1990. /ISBN 807011-056-2/.

6. Eckschlager, K. - Horsák, I. - Kodejš, Z.: Assessment of Analytical Results and Methods (in Czech), 1st edition, Praha, SNTL - Nakladatelství technické literatury 1980.

7. D'Agostino, R. B. - Belanger, A. - D'Agostino, Jr., R. B.: A Suggestion for Using Powerful and Informative Tests of Normality. The American Statistician, Vol. 44, No. 4, pp. 316-321, 1990.

\footnotetext{
${ }^{1}$ http://www $\cdot$ geotronics.cz/index $\cdot$ php?page=shop.product_details\&flypage=flypage .tpl\&product_ id=4\&category_id=15\&option=com_virtuemart\&Itemid=7
} 
